\title{
UNSTEADY FLOW OF MICROPOLAR MAXWELL FLUID OVER STRETCHING SURFACE
}

\author{
E.M.A. Elbashbeshy ${ }^{\mathrm{a}}$, K.M. Abdelgaber ${ }^{\mathrm{b}}$ and H.G. Asker $^{\mathrm{b}}$ \\ ${ }^{a}$ Mathematics Department, Faculty of Science, Ain Shams University, Abbassia, Cairo, Egypt. \\ ${ }^{\mathbf{b}}$ Department of Physics \& Engineering Mathematics, Faculty of Engineering - Mataria, Helwan University, \\ Cairo, Egypt.
}

Received 11/1/2018 Revised Accepted 26/3/2018

\begin{abstract}
The unsteady flow of Maxwell fluid over a stretching surface is investigated in this paper numerically. The governing partial differential equations are transformed into a nonlinear ordinary differential equations by using a similarity transformation. The numerical results are guaranteed in comparison with the previous under special assumptions. The effects of elasticity number and material parameter on velocity and micro-rotation profiles are presented and discussed in aid of tables and graphs.
\end{abstract}

Keywords: Maxwell Fluid, stretching surface, and micro-rotation

\section{Introduction}

Sakiadis' flow is an introductory work accomplished to detect the required force to pull a plate steadily through an incompressible Newtonian fluid. In real life, the most industrial fluids are nonNewtonian such as polymers melts, paints, lubricants, and suspended solutions. They are used in the production of adhesive taps, glass fiber, drawing of plastic sheets, etc. Figure 1 shows a melted substance extrudes through a die then it is stretched by wind roll-up while moving in a stationary fluid. The final mechanical properties of the product depend on the rate of cooling, drawing speed, and the properties of the cooling fluid such as thermal conductivity and viscosity. The non-Newtonian fluids branches into three categories; the differential type, rate type, and integral type. The Upper-Convected Maxwell (UCM) fluid is a viscoelastic fluid classified as rate type which takes into account the effect of the fluid elasticity on the boundary layer properties considering the characteristics of the relaxation time. Recently, a lot of numerical and analytical research analyzed the Maxwell fluid flow above a stretching surface. A lot of factors are considered such as magnetic parameter, Prandtl number, Deborah number, thermal heat transfer, etc.

Sadeghy et al. [1] introduced a simple analysis to predict the required force to pull a surface through a non-Newtonian fluid. Sadeghy's work stated that the fluid's elasticity destroys the similarity between the velocity profiles and lowers the drag force for large values of Deborah number (high Deborah number is close to reality). The flow and heat transfer of Maxwell fluid over a stretching 
surface are analyzed by Hayat et al. [2] presenting a series solution for the heat equation with convective boundary conditions. Singh and Agarwal [3] considered a variable viscosity and thermal conductivity over exponentially stretching surface.

Aliakbar et al. [4] mentioned that increasing the elasticity number and the radiation parameter increases the rate of cooling. Furthermore, they predicted that increasing Prandtl number causes a decrease in the fluid temperature in contrary with Eckert number. Subhas et al. [5] presented almost similar investigation for magneto-hydrodynamic (MHD) fluid.

\begin{tabular}{|c|c|c|}
\hline \multicolumn{3}{|c|}{ Nomenclature } \\
\hline \multicolumn{3}{|c|}{$f, g \quad$ Dimensionless functions } \\
\hline$j$ & The micro-inertia per unit mass & $v \quad$ Kinematical viscosity \\
\hline$K$ & The material parameter & $\rho \quad$ Fluid density \\
\hline$n, b$ & Constants & $\lambda$ The relaxation time of the fluid \\
\hline$N$ & The micro-rotation. & $\beta \quad$ The elasticity parameter \\
\hline$S$ & the vortex viscosity & $\gamma \quad$ The spin-gradient viscosity \\
\hline$t$ & Time & $\eta \quad$ Dimensionless coordinate \\
\hline$U_{w}$ & Velocity of solid surface & The stream function \\
\hline$u, v$ & Velocity components & Subscripts \\
\hline$x, y$ & Spatial coordinates & W Condition on the wall \\
\hline Greel & mbols & $\infty$ Free stream condition \\
\hline$\zeta$ & Unsteadiness parameter & \\
\hline
\end{tabular}

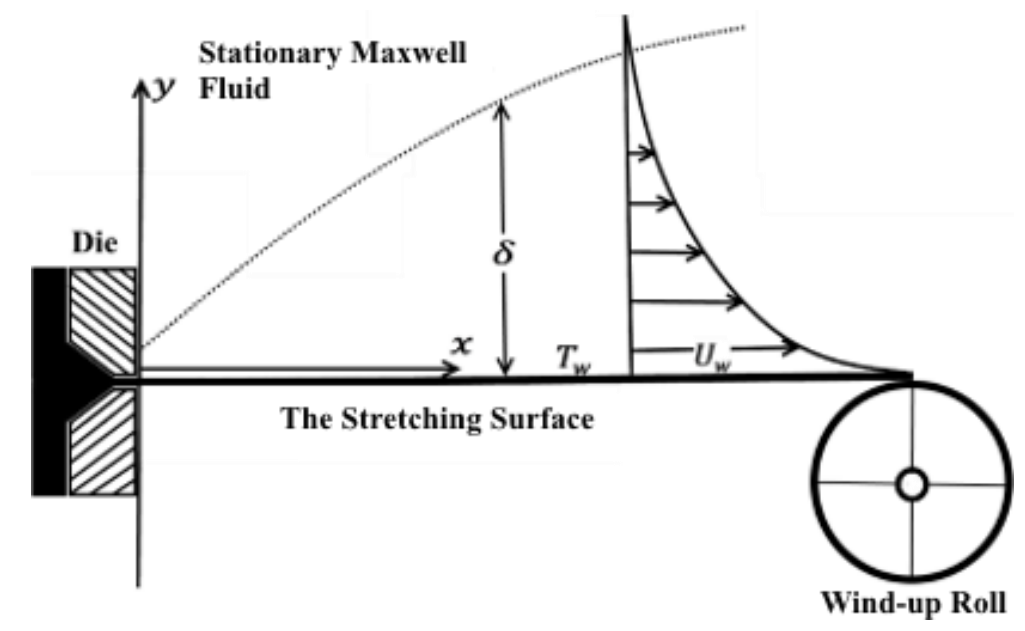

Fig. 1 schematic for flow above stretching surface

Alizadeh and Sadeghy [6] presented an analytical solution using homotopy technique for the unsteady MHD flow of Maxwell fluid above impulsively stretching surfaces which may be used as a comparator to check the performance of other numerical solutions.

The MHD Maxwell fluid is considered in a lot of research. Hayat and Qasim [7] studied the effects of thermophoresis and thermal radiation parameters. Furthermore, Shateyi [8] studied the thermophoresis effect in the presence of a chemical reaction in Darcian porous medium. Mukhopadhyay et al. [9] presented the effects of transpiration on the unsteady flow in the presence of 
first-order constructive/destructive chemical reaction. Nadeem et al. [10] considered the existence of the nanoparticle.

Hsiao [11] used the improved parameters control method to investigate the effects of the electrical MHD ohmic dissipation forced and free convection on the Maxwell fluid with a stagnation point. Furthermore, Hsiao [12] extended the previous study for a viscoelastic non-Newtonian Carreaunanofluid on a stagnation point.

On the other side, Eringen [13] introduced the theory of micropolar fluid which describes the inertial motion of the substructure particles. This phenomenon is related to the microscopic effects which in turn is related to the micro-rotation and the spin inertia of the suspended elements. All of these reveals the non-Newtonian behavior.

Aldawody and Elbashbeshy [14] studied the micropolar fluid considering the effects of the thermal and magnetic parameters. Then, Haque et al. [15] presented a numerical solution for the free convection and mass transfer in a porous medium with constant heat and mass flux. Furthermore, Qasim et al. [16] studied the effect of Newtonian heating. Hsiao [17] studied the effects of conjugate mixed convection, electrical magneto hydrodynamic, and heat source/sink on the nanofluid flow over a slip boundary stretching surface. Then, it was corrected in [18]. Furthermore, Hsiao [19] investigated the hydromagnetic flow with magnetic and viscous dissipation effects for a micropolar nanofluid over a stretching surface.

The heterogeneous reactions for the reactants of two or more phases and the homogeneous reactions for reactants of one phase take place while studying the stretching surfaces and cylinders. Hayat et al. [20] examined the thermally stratified mixed convection flow of an Oldroyd-B fluid and discussed the stagnation point flow towards a stretching surface. Hayat et al. [21] investigated same effects for viscoelastic fluid over a stretching cylinder with melting heat transfer. The fluid was electrically conducting through an inclined magnetic field. Hayat et al. [22] considered these effects with Newtonian heating in MHD flow of Powell-Eyring fluid by a stretching cylinder.

The analysis for single and multi-wall carbon nanotubes combined with the previous parameters is discussed by Hayat et al. [23]. Also, Hayat et al. [24] extended the investigation considering the stagnation point flow of viscous nanofluid towards a nonlinear stretching surface with variable thickness. Hayat et al. [25] presented an analysis dealt with a nanofluid flow due to a cylinder. Heat transfer mechanism was inspected under the physically acceptable convective type conditions.

The present work investigates the micropolar effect on the Maxwell fluid above stretching surface. The aim is to discuss the effects of the elasticity and the spin rotation on the Non-Newtonian fluids. The similarity transformation is used to obtain the non-dimensional ordinary equations which in turn are solved numerically. This solution considers the effects of the material parameter $K$ and the elasticity number $\beta$ on the velocity profile and the angular velocity of fluid rotation.

\section{Mathematical formulation}

Consider an incompressible, viscous, and unsteady state flow of non-Newtonian Maxwell fluid over a two dimensional stretching surface in the presence of a micropolar effect. The surface is stretched by the velocity $U_{w}=b x$ where $b$ is a constant. The surface is assumed to be impermeable, i.e., $v_{w}=0$. The $x$-axis is chosen along the stretching surface in the direction of the motion and $y$ axis is normal to it. The mathematical model governing this type of flow can be formulated as follows $[7,14]$ : 
$\frac{\partial u}{\partial x}+\frac{\partial v}{\partial y}=0$

$$
\begin{aligned}
\frac{\partial u}{\partial t}+u \frac{\partial u}{\partial x}+ & v \frac{\partial u}{\partial y}+\lambda\left[u^{2}\left(\frac{\partial^{2} u}{\partial x^{2}}\right)+v^{2}\left(\frac{\partial^{2} u}{\partial y^{2}}\right)+2 u v\left(\frac{\partial^{2} u}{\partial x \partial y}\right)\right] \\
& =\left(v+\frac{s}{\rho}\right) \frac{\partial^{2} u}{\partial y^{2}}+\frac{s}{\rho} \frac{\partial N}{\partial y}
\end{aligned}
$$

$\frac{\partial N}{\partial t}+u \frac{\partial N}{\partial x}+v \frac{\partial N}{\partial y}=\left(\frac{\gamma}{\rho j}\right) \frac{\partial^{2} N}{\partial y^{2}}-\frac{s}{\rho j}\left(2 N+\frac{\partial u}{\partial y}\right)$

subjected to boundary conditions

at $t=0: u(x, 0)=0, \quad v(x, 0)=0, \quad \lim _{y \rightarrow \infty} u(x, y)=0$

for $t>0: u(x, 0)=U_{w}, v(x, 0)=0, N(x, 0)=-n \frac{\partial u}{\partial y}, \lim _{y \rightarrow \infty} u(x, y)=\lim _{y \rightarrow \infty} v(x, y)=0$

Equation (1) introduces the continuity equation where $u$ and $v$ are the $x$ and $y$ components of the fluid velocity, respectively. Equation (2) introduces the $x$-momentum of the flow where the microrotation is $N$, the relaxation time parameter is $\lambda$, and the vortex viscosity is $s$. The density is $\rho$ and the dynamic viscosity is $v$ for the fluid. Equation (3) introduces the micro-rotation of the flow where the micro-inertia per unit mass is $j=v / b$, the spin gradient is $\gamma$, and $t$ is the time. The constant $n$ takes different values; $n=0$ for strong concentration and $n=0.5$ for weak concentration. The Maxwell fluid is viscous for $\lambda>0$ and $s>0$ while it is inviscid when $\lambda=s=0$.

The mathematical analysis of the problem is simplified by introducing the following dimensionless similarity variables

$\zeta=1-e^{-b t} ; \quad \eta=\sqrt{\frac{b}{v \zeta}} y ; \quad \psi=\sqrt{b v \zeta} x f(\eta) ; \quad N=b \sqrt{\frac{b}{v \zeta}} x g(\eta)$

to convert the partial differential equations into nonlinear ordinary differential equations where $\zeta$ is the unsteadiness parameter, $\eta$ is a dimensionless variable, and $\psi$ is the stream function. The dimensionless functions are $f$ and $g$. The continuity equation (1) is satisfied by assuming

$$
u=\frac{\partial \psi}{\partial y}=b x f^{\prime}(\eta), \quad v=-\frac{\partial \psi}{\partial x}=-\sqrt{b v \zeta} f(\eta)
$$

where prime denotes the differentiation with respect to $\eta$. Upon using equations (6-7), the governing equations are reduced into the dimensionless form

$$
\begin{aligned}
& (1+K) f^{\prime \prime \prime}+\frac{1}{2}(1-\zeta) \eta f^{\prime \prime}+\zeta\left(f f^{\prime \prime}-f^{\prime 2}\right)+\zeta \beta\left(2 f f^{\prime} f^{\prime \prime}-f^{2} f^{\prime \prime \prime}\right)+K g^{\prime}=0 \\
& \left(1+\frac{K}{2}\right) g^{\prime \prime}+\frac{1}{2}(1-\zeta)\left(g+\eta g^{\prime}\right)+\zeta\left(f g^{\prime}-f^{\prime} g\right)-K \zeta\left(2 g+f^{\prime \prime}\right)=0 \\
& f(0)=0 ; \quad f^{\prime}(0)=1 ; \quad g(0)=-n f^{\prime \prime}(0)
\end{aligned}
$$


$\lim _{\eta \rightarrow \infty} f^{\prime}(\eta)=0 ; \quad \lim _{\eta \rightarrow \infty} g(\eta)=0$

where the elasticity number is $\beta=b \lambda$ and the material parameter is $K=s / \rho v=s / \mu$. The skin friction coefficient takes the form

$$
C_{f}=\frac{\tau_{w}}{\rho U^{2} / 2}=\left[(\mu+\lambda b+s) \frac{\partial u}{\partial y}+s N\right]_{\text {at } y=0}
$$

where $\tau_{w}$ is the skin friction and the modified-skin friction is

$$
C_{f} \sqrt{R e_{x}}=-\zeta^{-0.5}[1+\beta+(1-n) K] f^{\prime \prime}(0)
$$

where the local Reynolds number is

$R e_{x}=\frac{\rho U_{w} x}{\mu}$

\section{Numerical solution}

The nonlinear differential equations (8-9) subjected to the boundary conditions (10-11) are converted into the following simultaneous system of first order differential equations as follows:

$y_{1}^{\prime}=y_{2}$

$y_{2}^{\prime}=y_{3}$

$y_{3}^{\prime}=\frac{0.5 \eta(\zeta-1) y_{3}+\zeta\left(y_{2}^{2}-y_{1} y_{3}+M y_{3}\right)-2 \zeta \beta y_{1} y_{2} y_{3}-K y_{5}}{\left(1+K-\zeta \beta y_{1}^{2}\right)}$

$y_{4}^{\prime}=y_{5}$

$y_{5}^{\prime}=\frac{0.5(\zeta-1)\left(y_{4}+\eta y_{5}\right)+\zeta\left(y_{2} y_{4}-y_{1} y_{5}\right)+K \zeta\left(2 y_{4}+y_{3}\right)}{(1+K / 2)}$

where $y_{1}=f, y_{2}=f^{\prime}, y_{3}=f^{\prime \prime}, y_{4}=g$, and $y_{5}=g^{\prime}$. The initial conditions are

$y_{1}(0)=0, y_{2}(0)=1, y_{3}(0)=s_{1}, y_{4}(0)=-n y_{3}(0), y_{5}(0)=s_{2}$

where $s_{1}$ and $s_{2}$ are priori unknowns to be determined as a part of the solution.

The system of equations (15-19) subjected to the initial conditions (20) are solved by fourth/fifth order Runge-Kutta method in combination with shooting method as used and explained by Elbashbeshy et al. [27].

\section{Results and discussion}

Tables 1-3 present a comparison with others. The tables show an excellent agreement for different settings. Table 1 presents the results for the modified skin friction at $\beta=0$ considering the steady state $(\zeta=1)$ and strong concentration $(n=0)$ in comparison with Aldawody and Elbashbeshy [14], Qasim et al. [16], and Ishak et al. [28]. Table 2 considers a weak concentration $(n=0.5)$. Finally, Table 3 presents a comparison for $-f^{\prime \prime}(0)$ with Subahs et al. [5], Swati et al. [9], Hsiao [19], and Sadeghy et al. [29]. 
Table 4 introduces a tabulated data for $-f^{\prime \prime}(0)$ and the modified skin friction at different settings. The first part in Table 4 shows the effect of the material parameter $K$ at $\beta=0$ with strong concentration $(n=0)$. The value of $-f^{\prime \prime}(0)$ decreases while the modified skin friction increases by increasing the values of $K$ which explains the increase in the shear stress. Part 2 of Table 4 presents the same response for weak concentration $(n=0.5)$. By comparing parts 1 and 2 , the modified skin friction for strong concentration is a little higher than for weak concentration. Part 3 in Table 4 introduces the previous settings in part 1 considering the Maxwell fluid $(\beta=0.2)$. It shows a decrease in $-f^{\prime \prime}(0)$ and an increase in the modified skin friction.

Table 1. Comparison of $C_{f} \sqrt{R e_{x}}$ with others at $\beta=0, \zeta=1$, and $n=0$ for different values of $K$.

\begin{tabular}{|l|llll|}
\cline { 2 - 5 } \multicolumn{1}{c|}{} & \multicolumn{4}{c|}{ Modified Skin Friction $\boldsymbol{C}_{\boldsymbol{f}} \sqrt{\boldsymbol{R e}_{\boldsymbol{x}}}$} \\
\hline $\boldsymbol{K}$ & Aldawody [14] & Qasim [16] & Ishak [28] & Present results \\
\hline 0 & 1 & 1 & 1 & 0.999999 \\
\hline 1 & 1.3679 & 1.367872 & 1.3679 & 1.36793 \\
2 & 1.6213 & 1.621225 & 1.6213 & 1.62133 \\
4 & 2.0043 & 2.004133 & 2.0042 & 2.0054 \\
\hline
\end{tabular}

Table 2. Comparison of $\boldsymbol{C}_{f} \sqrt{\boldsymbol{R} \boldsymbol{e}_{\boldsymbol{x}}}$ with others at $\boldsymbol{\beta}=\mathbf{0}, \zeta=\mathbf{1}$, and $\boldsymbol{n}=\mathbf{0 . 5}$ for different values of $\boldsymbol{K}$.

\begin{tabular}{|l|llll|}
\cline { 2 - 5 } \multicolumn{1}{c|}{} & \multicolumn{4}{c|}{ Modified Skin Friction $C_{f} \sqrt{R e_{x}}$} \\
\hline $\boldsymbol{K}$ & Aldawody [14] & Qasim [16] & Ishak [28] & Present results \\
\hline 0 & 1 & 1 & 1 & 0.99999 \\
1 & 1.2247 & 1.224741 & 1.2247 & 1.22463 \\
\hline 2 & 1.4142 & 1.414218 & 1.4142 & 1.41421 \\
4 & 1.7343 & 1.732052 & 1.7343 & 1.73205 \\
\hline
\end{tabular}

Table 3. Comparison of $-\boldsymbol{f}^{\prime \prime}(\mathbf{0})$ with others at $\boldsymbol{\zeta}=\mathbf{1}$ and $\boldsymbol{n}=\mathbf{0}$ for different values of $\boldsymbol{\beta}$ and $\boldsymbol{K}$.

\begin{tabular}{|c|c|c|c|c|c|c|}
\hline $\boldsymbol{K}$ & $\beta$ & $\begin{array}{l}\text { Subahs et al. } \\
{[5]}\end{array}$ & $\begin{array}{l}\text { Swati et al. } \\
{[9]}\end{array}$ & Hsiao [19] & $\begin{array}{l}\text { Sadeghy et al. } \\
\text { [29] }\end{array}$ & Present results \\
\hline \multirow{8}{*}{0} & 0 & 0.999962 & 0.999963 & & 1 & 0.999999999 \\
\hline & 0.2 & 1.051948 & 1.051949 & & 1.0549 & 1.051889896 \\
\hline & 0.4 & 1.101850 & 1.101851 & & 1.10084 & 1.101903319 \\
\hline & 0.6 & 1.150163 & 1.150162 & & 1.15016 & 1.150137398 \\
\hline & 0.8 & 1.196692 & 1.196693 & & 1.19872 & 1.196711332 \\
\hline & 1.2 & 1.285257 & & & & 1.285363315 \\
\hline & 1.6 & 1.368641 & & & & 1.368758413 \\
\hline & 2 & 1.447617 & & & & 1.447650711 \\
\hline 0.2 & 0 & & & 0.9098 & & 0.909750320 \\
\hline
\end{tabular}

For high elasticity parameter $\beta$, the viscosity and resistivity of the fluid are increased inducing more friction within the fluid and reducing the velocity of the flow. These effects appear in the increasing of the modified skin friction and $-f^{\prime \prime}(0)$ as tabulated in parts 4 and 5 of Table 4 for strong and weak concentration (neglecting the micropolar effect $K=0$ ), respectively. The two previous parts show that the concentration has no effect on the modified 
skin friction if $K=0$. Part 6 of Table 4 shows the effect of $\beta$ in combination with micropolar effect $(K=2)$. It presents an increase in the modified skin friction in comparison with part 4.

The last two parts of Table 4 present the effect of $\zeta$ for the Maxwell fluid $(\beta=0.4)$ considering the micropolar effect $(K=2)$ on the modified skin friction. The modified skin friction decreases with increasing the unsteadiness parameter $\zeta$. The weak concentration enhances the decrease of the modified skin friction in comparison with the strong one.

The material parameter $K$ presents the vortex viscosity. This type of viscosity relates the average shear stress in the flow to the vertical gradient of the velocity. Figures 2 and 3 present the influences of the material parameter on the velocity profile at $\beta=0$ for strong and weak concentrations, respectively. The velocity profile increases with increasing the material parameter.

Figures 4 and 5 present the effects of the material parameter $K$ on the micro-rotation profile. For strong concentration $(n=0)$, Figure 4 shows an increase in the micro-rotation profile. On the other hand, Figure 5 shows that the micro-rotation profile decreases as $K$ increases if $\eta<$ 1.3 while the reverse process takes place if $\eta>1.3$.

Figures 6 and 7 show the effects of elasticity parameter $\beta$ on the velocity profile in the absence of micropolar effect $(K=0)$. The two figures show a decrease in the velocity with increasing the elasticity number. Physically, the elasticity number increases the resistance within the fluid then the velocity decreases. At a high elasticity number, the material behavior approaches the solid behavior which in turn slows down the flow velocity. Furthermore, Figures 8 and 9 present a decrease in the velocity with increasing the elasticity parameter in the existence of micropolar effect. In comparison between Figures (6-7) and Figures (8-9), the micropolar effect decreases the effect of $\beta$ on the velocity.

Table 4. The modified skin-friction at different values of $n, \beta, K$, and $\zeta$

\begin{tabular}{lccccc} 
Concentration & $\zeta$ & $K$ & $\beta$ & $-f^{\prime \prime}(0)$ & $C_{f} \sqrt{R e_{x}}$ \\
\hline Strong $n=0$ & 1 & 0 & 0 & 1 & 1 \\
& & 1 & & 0.683965 & 1.367930 \\
\hline Weak $n=0.5$ & 1 & 0 & 0 & 0.540446 & 1.621338 \\
\hline & & 1 & & 0.816382 & 1 \\
\hline Strong $n=0$ & 1 & 0 & 0.2 & 1.051889 & 1.224573 \\
\hline & & 1 & & 0.720763 & 1.2622668 \\
\hline Strong $n=0$ & 1 & 0 & 0.4 & 1.10903 & 1.5856786 \\
\hline & & & 0.6 & 1.150137 & 1.5249344 \\
\hline Weak $n=0.5$ & 1 & 0 & 0.4 & 1.101903 & 1.8402192 \\
\hline & & & 0.6 & 1.150137 & 1.5426642 \\
\hline
\end{tabular}




\begin{tabular}{lccccc}
\hline Strong $n=0$ & & & 0.6 & 0.626929 & 2.2569444 \\
\hline \multirow{3}{*}{ Strong $n=0$} & & 0.8 & 0.653857 & 2.4846566 \\
\hline \multirow{3}{*}{ Weak $n=0.5$} & 0.4 & 2 & 0.4 & 0.448511 & 2.41113774 \\
& 0.8 & & & 0.553371 & 2.10353779 \\
\cline { 2 - 6 } & 1 & & & 0.599089 & 2.0369026 \\
\hline & 0.4 & 2 & 0.4 & 0.581557 & 2.20685365 \\
\hline
\end{tabular}
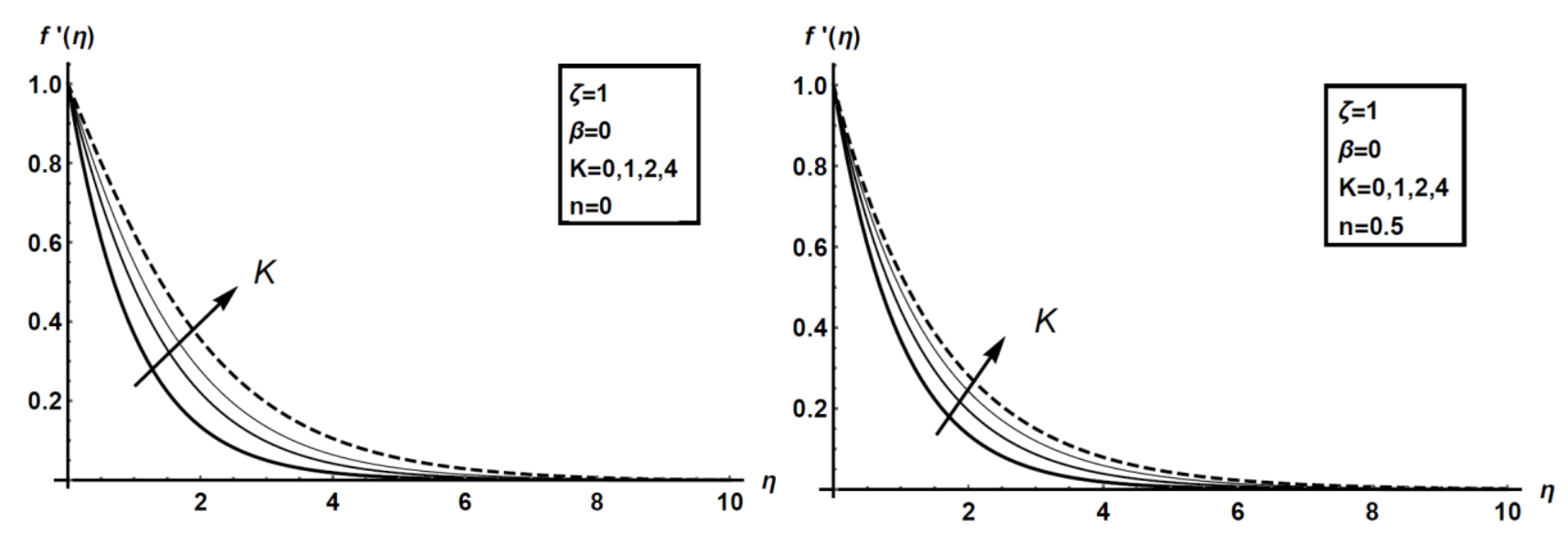

Fig. 2. The effect of $K$ on the velocity at $\beta=0$, Fig. 3. The effect of $K$ on the velocity at $\beta=0$, $n=0$, and $\zeta=1$ $n=0.5$, and $\zeta=1$
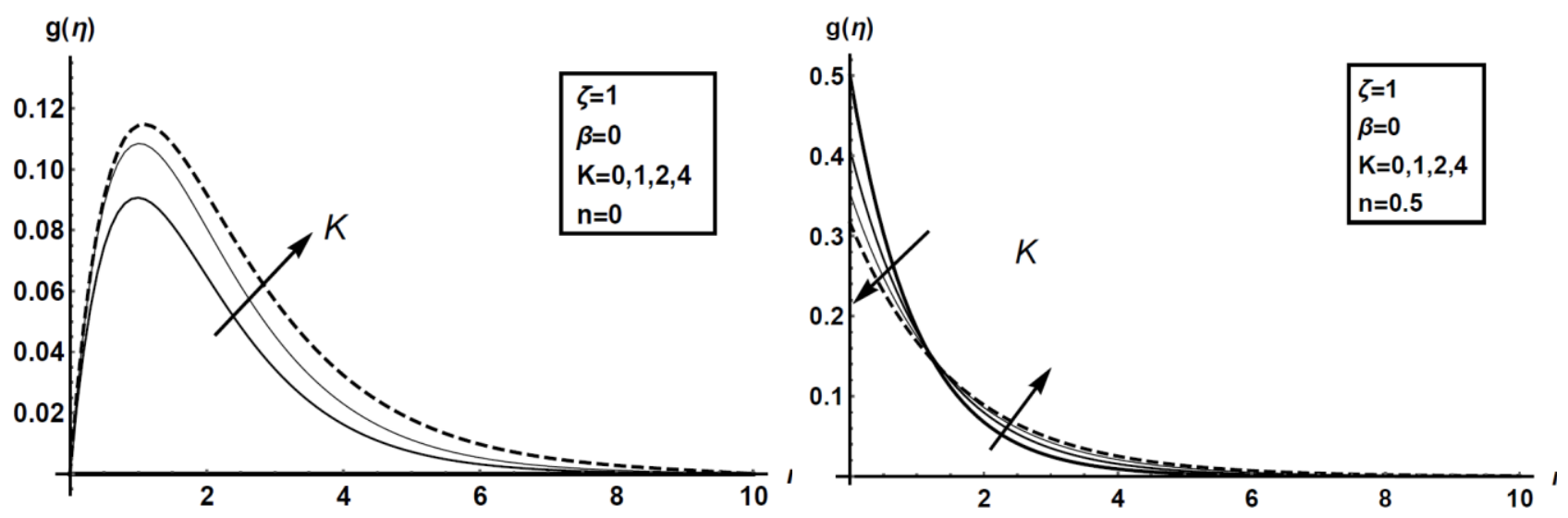

Fig. 4. The effect of $K$ on the micro-rotation velocity at $\beta=0, n=0$, and $\zeta=1$

Fig. 5. The effect of $K$ on the micro-rotation velocity at $\beta=0, n=0.5$, and $\zeta=1$ 

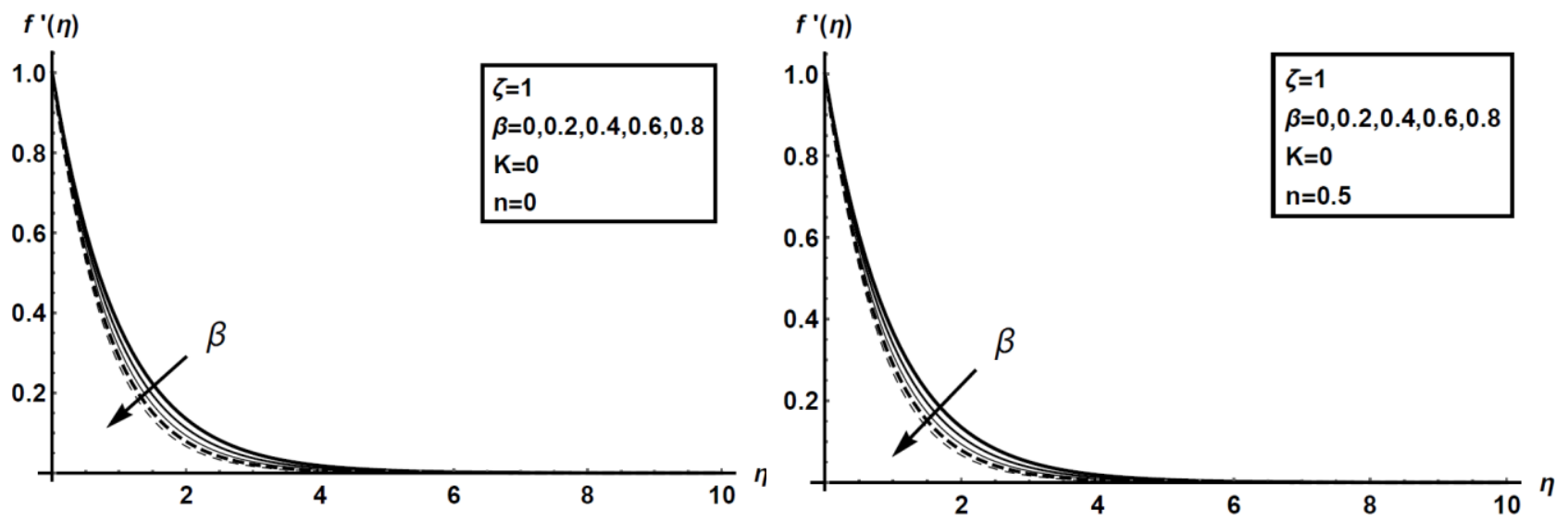

Fig. 6. The effect of $\beta$ on the velocity profile at $K=0, n=0$, and $\zeta=1$

Fig. 7. The effect of $\beta$ on the velocity profile at $K=0, n=0.5$, and $\zeta=1$
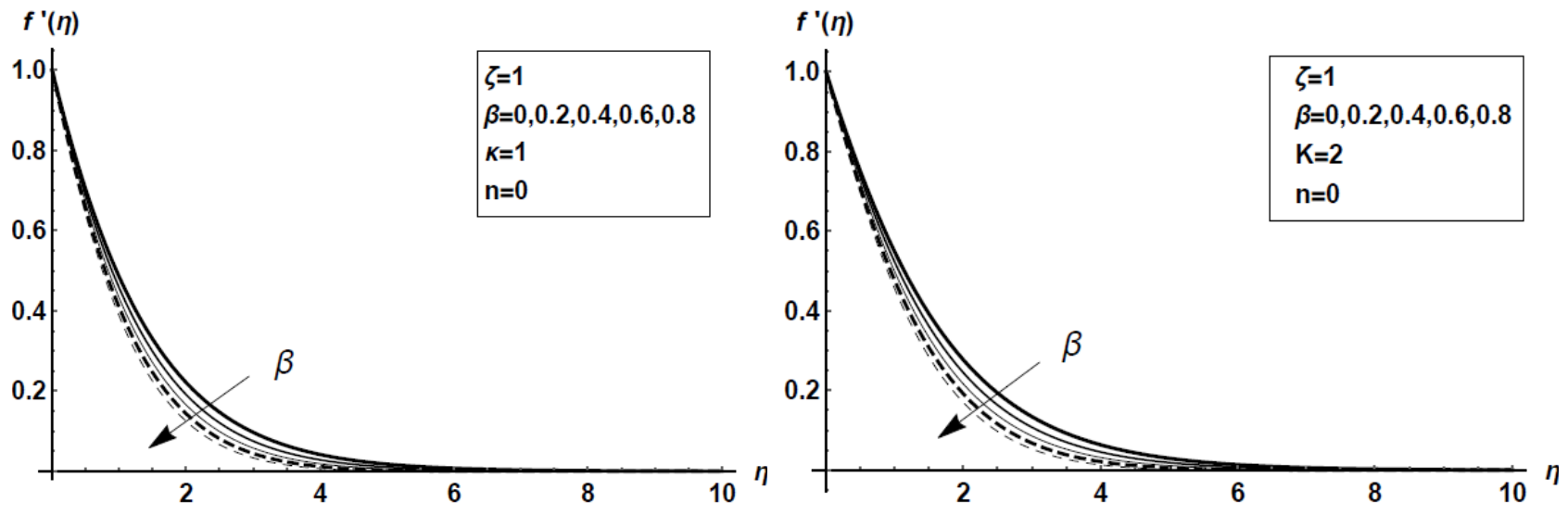

Fig. 8. The effect of $\beta$ on the velocity profile at $K=1, n=0$, and $\zeta=1$

Fig. 9. The effect of $\beta$ on the velocity profile at $K=2, n=0$, and $\zeta=1$

Figures 10-12 present the effects of the elasticity parameter on the micro-rotation profile. Figure 10 shows a slight increase in the maximum value of the angular velocity at $K=0$ for weak concentration $(n=0.5)$. Figures 11 and 12 show that the increase of micro-rotation profile inverses at $\eta=1.75$ for strong concentration $(n=0)$ in the presence of micropolar effect $(K=1$ and $K=2)$. 

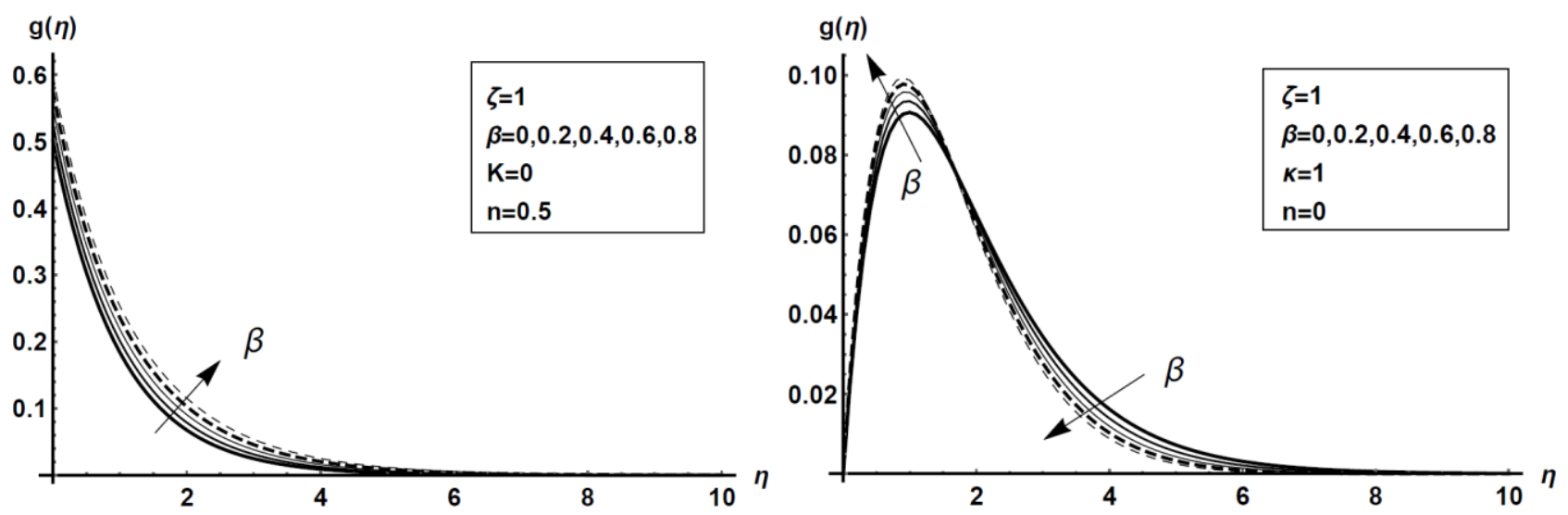

Fig. 10. The effect of $\beta$ on the micro-rotation profile at $K=0, n=0.5$, and $\zeta=1$

Fig. 11. The effect of $\beta$ on the micro-rotation profile at $K=1, n=0$, and $\zeta=1$

Figures 13-14 present the increase of velocity profile by increasing the material parameter for the Maxwell fluid at $\beta=0.1$ and 0.2 , respectively. Figures $15-16$ present the increase in the microrotation profile with increasing the material parameter for the Maxwell fluid at $\beta=0.1$ and 0.2 , respectively.

By increasing the unsteadiness parameter $\zeta$, Figures 17 and 20 show a decrease in the velocity profile and an increase in the micro-rotation profile, respectively; while reaching the steady-state flow for strong concentration $(n=0)$. From Figure 18, the velocity profile is decreased for weak concentration. As shown in Figure 20, the micro-rotation profile is increased if $\eta<0.5$ but it is reversed if $\eta>0.5$ for weak concentration $(n=0.5)$.

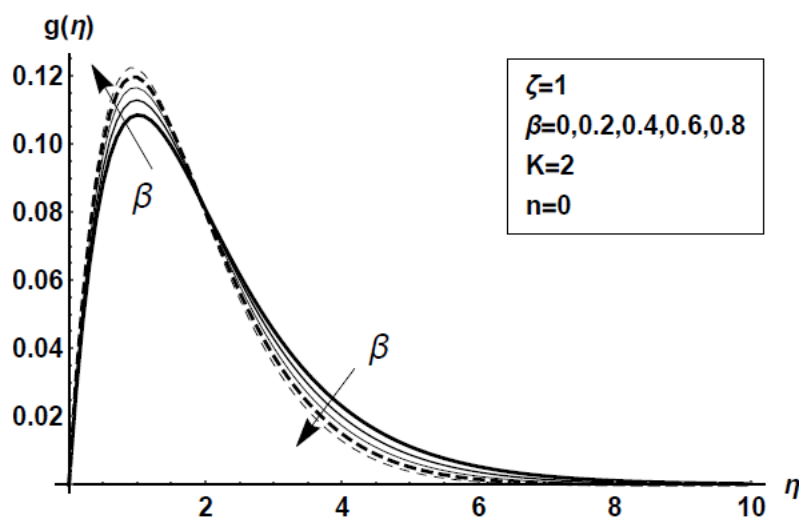

Fig. 12. The effect of $\beta$ on the micro-rotation profile at $K=2, n=0$, and $\zeta=1$

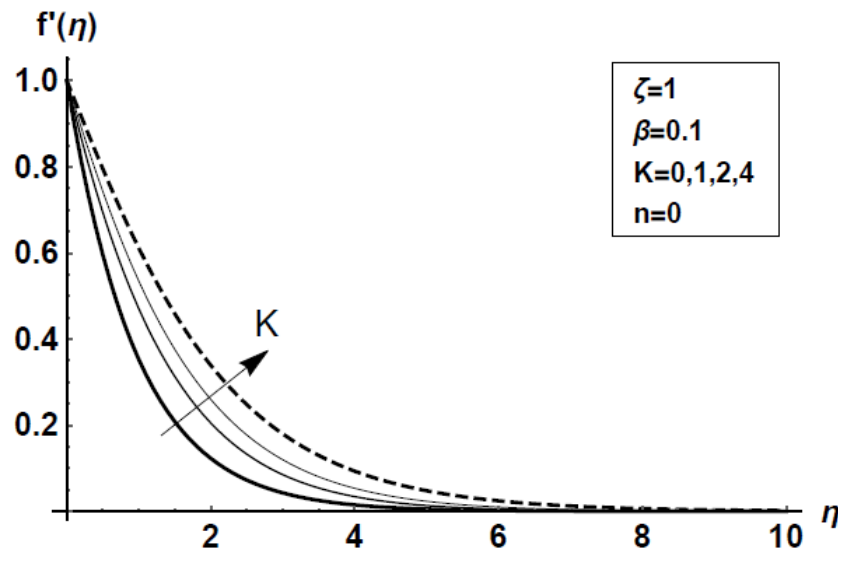

Fig. 13. The effect of $K$ on the velocity profile at $\beta=0.1, n=0$, and $\zeta=1$ 


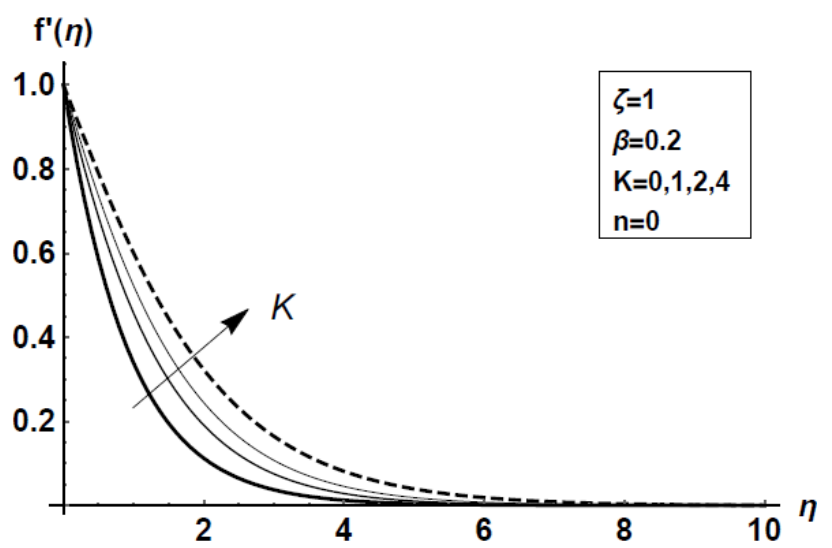

Fig. 14. The effect of $K$ on the velocity at $\beta=$ $0.2, n=0$, and $\zeta=1$

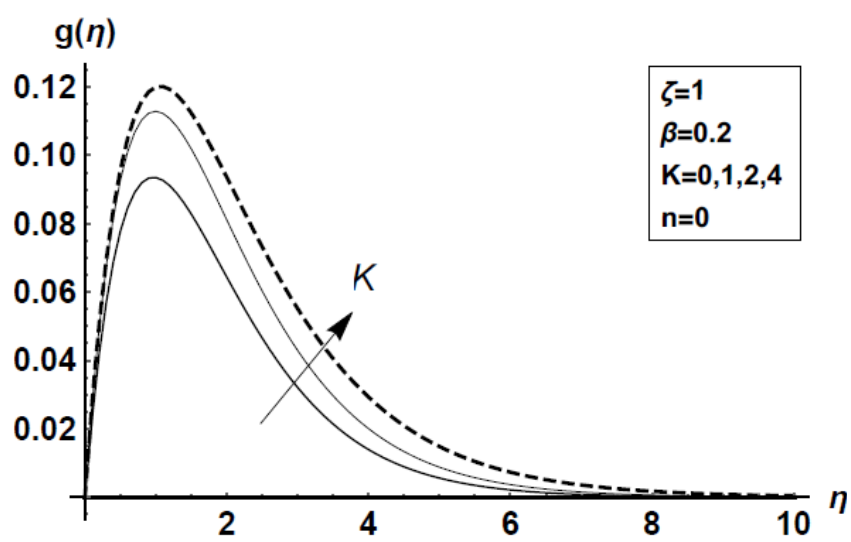

Fig. 16. The effect of $K$ on the micro-rotation velocity at $\beta=0.2, n=0$, and $\zeta=1$

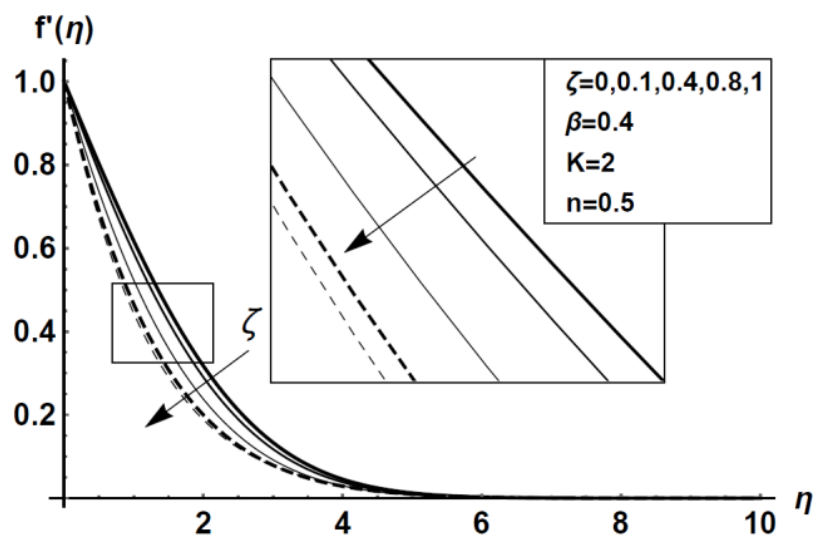

Fig. 18. The effect of $\zeta$ on the velocity profile at $K=2, n=0.5$, and $\beta=0.4$

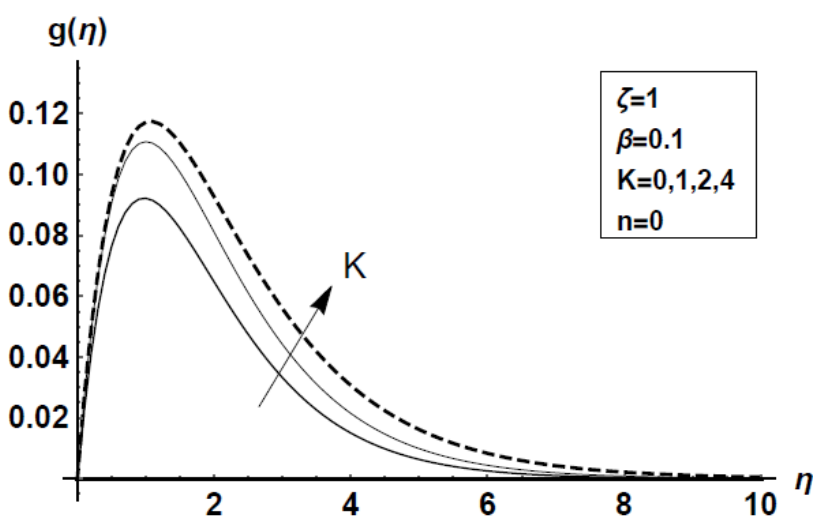

Fig. 15. The effect of $K$ on the micro-rotation profile at $\beta=0.1, n=0$, and $\zeta=1$

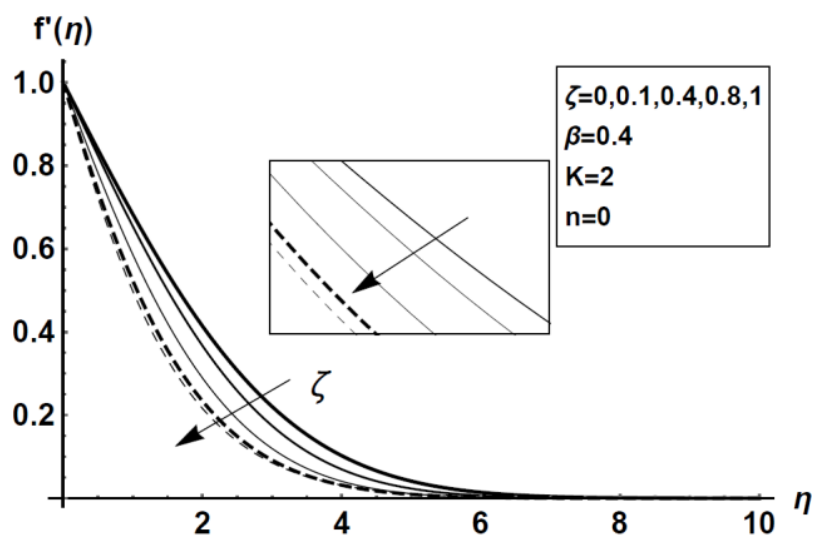

Fig. 17. The effect of $\zeta$ on the velocity profile at $K=2, n=0$, and $\beta=0.4$

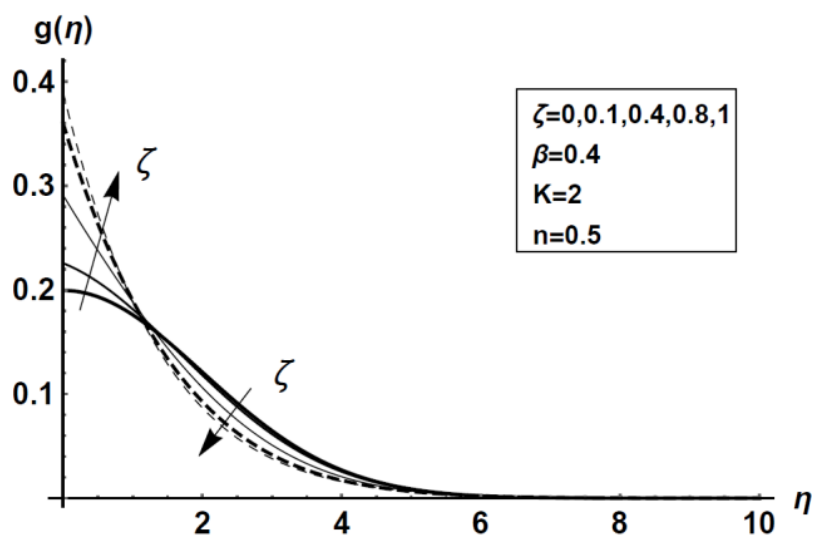

Fig. 19. The effect of $\zeta$ on the micro-rotation profile at $K=2, n=0.5$, and $\beta=0.4$ 


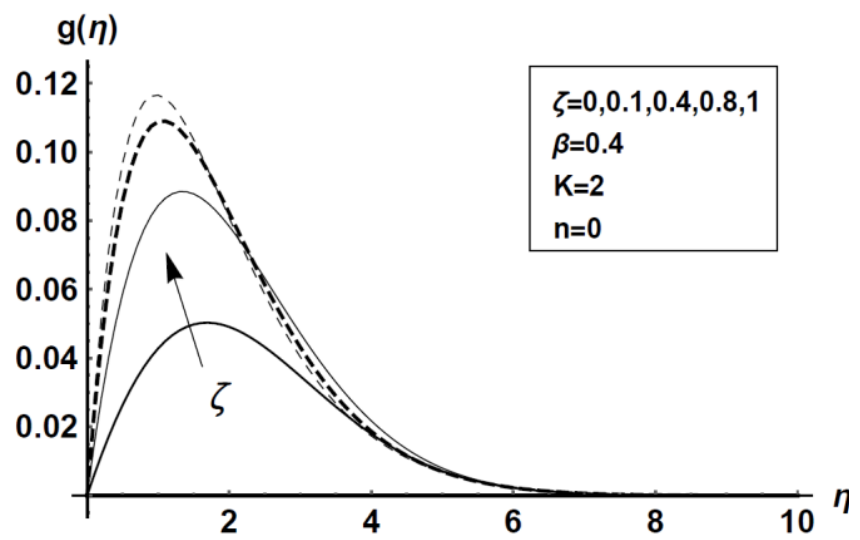

Fig. 20. The effect of $\zeta$ on the micro-rotation profile at $K=2, n=0$, and $\beta=0.4$

\section{Conclusions}

This dissertation can be considered as a numerical treatment for the boundary-layer problem for the Maxwell fluid considering the micro-rotation effect. An excellent agreement with the previous results for Micropolar fluids and Maxwell fluids is achieved. The numerical results are presented graphically, and the local skin friction also is investigated at different settings. Finally The observations of the present study can be summarized in the following points.

1- Increasing the elasticity number produces a drag force in the flow. Then, the velocity decreases while the maximum value of the angular velocity increases.

2- The effect of the material parameter in increasing the horizontal velocity and the maximum value of the angular velocity is obvious for the Maxwell fluid. This effect is equal either for strong or weak concentration. Thus, the shear stress increases.

3- The skin friction increases as the material parameter decreases and the elasticity number increases.

\section{Acknowledgement}

The authors wish to express their cordial thanks to the Editor and the referees for their helpful comments and suggestions

\section{References}

1. K. Sadeghy, A.H. Najafi, and M. Saffaripour, Sakiadis flow of an upper-convected Maxwell fluid, International Journal of Non-Linear Mechanics. 40, pp. 1220-1228, 2005.

2. T. Hayat, B. S.A. Shehzada, M. Qasima, and S. Obaidat, Steady Flow of Maxwell Fluid with Convective Boundary Conditions, Zeitschrift für Naturforschung A, 66, pp. 417-422, 2011.

3. V. Singh and Sh. Agarwal, Flow and Heat Transfer of Maxwell Fluid with Variable Viscosity and Thermal Conductivity over an Exponentially Stretching Sheet, American Journal of Fluid Dynamics, 3(4), pp. 87-95, 2013. 
4. V. Aliakbar, A. Alizadeh-Pahlavan, and K. Sadeghy, The influence of thermal radiation on MHD flowof Maxwellian fluids above stretching sheets, Communications in Nonlinear Science and Numerical Simulation, 14, pp. 779-794, 2009.

5. M.S. Abel, J.V. Tawade, and M.M. Nandeppanavar, MHD flow and heat transfer for the upper-convected Maxwell fluid over a stretching sheet, Meccanica, 47, pp. 385-393, 2012.

6. A. Alizadeh-Pahlavan and K. Sadeghy, On the use of homotopy analysis method for solving unsteady MHD flow of Maxwellian fluids above impulsively stretching sheets, Commun. Nonlinear Sci Numer Simulat., 14, pp. 1355-1365, 2009.

7. T. Hayat and M. Qasim, Influence of thermal radiation and Joule heating on MHD flow of a Maxwell fluid in the presence of thermophoresis, International Journal of Heat and Mass Transfer, 53, pp. 4780-4788, 2010.

8. S. Shateyi, A new numerical approach to MHD flow of a Maxwell fluid past a vertical stretching sheet in the presence of thermophoresis and chemical reaction, Boundary Value Problems, 196, 2013.

9. S. Mukhopadhyay, M.G. Arif, and M.W.A. Pk, Effects of transpiration on unsteady MHD flow of an upper convected Maxwell (UCM) fluid passing through a stretching surface in the presence of a first order chemical reaction, Chin. Phys., 22 (12), 2013.

10. S. Nadeem, R.U. Haq, and Z.H. Khan, Numerical study of MHD boundary layer flow of a Maxwell fluid past a stretching sheet in the presence of nanoparticles, Journal of the Taiwan Institute of Chemical Engineers, 2013.

11 K.L. Hsiao, Combined Electrical MHD Heat Transfer Thermal Extrusion System Using Maxwell Fluid with Radiative and Viscous Dissipation Effects, Applied Thermal Engineering, 2016. DOI: 10.1016/j.applthermaleng.2016.08.208.

12 K.L. Hsiao, To Promote Radiation Electrical MHD Activation Energy Thermal Extrusion Manufacturing System Efficiency by Using Carreau-Nanofluid with Parameters Control Method, Energy, 130, pp. 486-499, 2017.

13. Eringen, Theory of Micropolar fluid, J. math mech., 16, pp. 1-18, 1966.

14. D.A. Aldawody and E.M.A. Elbashbeshy, Heat transfer over an unsteady stretching surface in a micropolar fluid in the presence of magnetic field and thermal radiation, Can. J. phys., 89, pp. 295-298, 2011.

15. Md.Z. Haque, Md.M. Alam, M. Ferdows, and A. Postelnicu, Micropolar fluid behaviors on steady MHD free convection and mass transfer flow with constant heat and mass fluxes, joule heating and viscous dissipation, Journal of King Saud University - Engineering Sciences, 24, pp. 71-84, 2012.

16. M. Qasim, I. Khan, and Sh. Shafie, Heat Transfer in a Micropolar Fluid over a Stretching Sheet with Newtonian Heating, PLOS ONE, 8 (4), 2013.

17 K.L. Hsiao, Stagnation Electrical MHD Nanofluid Mixed Convection with Slip Boundary on a Stretching Sheet, Applied Thermal Engineering, 2016. doi:10.1016/j.applthermaleng.2015.12.138, 98, 850-861

18 K.L. Hsiao, Corrigendum to "Stagnation Electrical MHD Nanofluid Mixed Convection with Slip Boundary on a Stretching Sheet" [Applied Thermal Engineering, 98 (2016)850-861], Applied Thermal Engineering, 2017. doi.org/10.1016/j.applthermaleng. 2017. 07.170

19 K.L. Hsiao, Micropolar Nanofluid Flow with MHD and Viscous Dissipation Effects Towards a Stretching Sheet with Multimedia Feature, International Journal of Heat and Mass Transfer, 112, pp. 983-990, 2017. 
20 T. Hayat, Z. Hussain, M. Farooq, A. Alsaedi, and M. Obaid, Thermally Stratified Stagnation Point Flow of an Oldroyd-B Fluid, Int. J. Nonlinear Sci. Numer. Simul., 15(1), pp. 77-86, 2014.

21 T. Hayat, Z. Hussain, M. Farooq, and A. Alsaedi, Effects of homogeneous and heterogeneous reactions and melting heat in the viscoelastic fluid flow, Journal of Molecular Liquids, 215, pp. 749-755, 2016.

22 T. Hayat, Z. Hussain, A. Alsaedi, and M. Farooq, Magnetohydrodynamic Flow by a Stretching Cylinder with Newtonian Heating and Homogeneous-Heterogeneous Reactions, PLOS ONE, 2016. DOI:10.1371/journal.pone.0156955.

23 T. Hayat, Z. Hussain, A. Alsaedi, and B. Ahmad, Heterogeneous-homogeneous reactions and melting heat transfer effects in flow with carbon nanotubes, Journal of Molecular Liquids, 220, pp. 200-207, 2016.

24 T. Hayat, Z. Hussain, A. Alsaedi, and S. Asghar, Carbon nanotubes effects in the stagnation point flow towards a nonlinear stretching sheet with variable thickness, Advanced Powder Technology, 2016.

25 T. Hayat, Z. Hussain, A. Alsaedi, and M. Mustafa, Nanofluid flow through a porous space with convective conditions and heterogeneous-homogeneous reactions, Journal of the Taiwan Institute of Chemical Engineers, 70, pp. 119-126, 2017.

26. E.M.A. Elbashbeshy, T.G. Emam, M.S. El-Azab and K.M. Abdelgaber, Laminar boundary layer flow along a stretching cylinder embedded in a porous medium, International Journal of the Physical Sciences, 7(24), pp. 3067-3072, 2012.

27. E.M.A. Elbashbeshy, T.G. Emam, M.S. El-Azab, and K.M. Abdelgaber, Effect of thermal radiation on flow, heat, and mass transfer of a Nanofluid over a stretching horizontal cylinder embedded in a porous medium with suction/injection, Journal of Porous Media, 18(3), pp. 215-229, 2015.

28. A. Ishak, R. Nazar, and I. Pop, Hydromagnetic flow and heat transfer adjacent to a stretching vertical sheet, Heat Mass Transfer, 44, pp. 921-927, 2008.

29. K. Sadeghy, H. Hajibeygi, and S.M. Taghavi, Stagnation point flow of upper-convected Maxwell fluids, Int. J. Non-Linear Mech., 41, pp. 1242-1247, 2006. 